\title{
Concurrent use of metformin enhances the efficacy of EGFR-TKIs in patients with advanced EGFR-mutant non-small cell lung cancer-an option for overcoming EGFR-TKI resistance
}

\author{
Ruoshuang Han ${ }^{1 \#}$, Yijun Jia ${ }^{1 \#}$, Xuefei $\mathrm{Li}^{2}$, Chao Zhao ${ }^{2}$, Sha Zhao ${ }^{1}$, Sangtian Liu ${ }^{1}$, Yiwei Liu ${ }^{1}$, \\ Donglai Chen ${ }^{3}$, Qian Zhang ${ }^{1}$, Xiaozhen Liu ${ }^{1}$, Jinpeng Shi ${ }^{1}$, Jiayu Li ${ }^{1}$, Caicun Zhou ${ }^{1}$ \\ ${ }^{1}$ Department of Medical Oncology, Shanghai Pulmonary Hospital, Tongji University School of Medicine, Shanghai, China; ${ }^{2}$ Department of Lung \\ Cancer and Immunology, Shanghai Pulmonary Hospital, Tongji University School of Medicine, Shanghai, China; ${ }^{3}$ Department of Thoracic Surgery, \\ Shanghai Pulmonary Hospital, Tongji University School of Medicine, Shanghai, China \\ Contributions: (I) Conception and design: R Han; Y Jia; (II) Administrative support: C Zhou; J Li; (III) Provision of study materials or patients: X \\ Li, C Zhao, D Chen; (IV) Collection and assembly of data: Q Zhang, S Zhao, X Liu; (V) Data analysis and interpretation: S Liu; Y Liu; J Shi; (VI) \\ Manuscript writing: All authors; (VII) Final approval of manuscript: All authors. \\ \#These authors contributed equally to this work. \\ Correspondence to: Caicun Zhou, MD, PhD. No. 507, Zheng Min Road, Shanghai, 200433, China. Email: doctorcaicunzhou@163.com.
}

Background: Resistance is almost inevitable and is still a major obstacle in epidermal growth factor receptor (EGFR)-tyrosine kinase inhibitor (TKI) therapy. Only limited relevant clinical studies evaluated the therapeutic effects by combing metformin and EGFR-TKIs in non-small cell lung cancer (NSCLC) patients. Therefore, we evaluated the efficacy of concurrent use of metformin with EGFR-TKIs, and assessed whether the addition of metformin may improve clinical outcomes and delay the occurrence of EGFR-TKI resistance.

Methods: We conducted cell proliferation and apoptosis assay for investigation of metformin in combination with EGFR-TKIs to overcome EGFR-TKI resistance in vitro. Furthermore, we retrospectively reviewed clinicopathological characteristics and therapeutic outcomes of EGFR-mutant advanced NSCLC diabetic patients who received EGFR-TKIs with or without concurrent use of metformin.

Results: In vitro experiment, metformin showed synergistic interaction both with gefitinib in PC9R (CI $=0.77)$ and with osimertinib in PC9R/OR $(\mathrm{CI}=0.77)$ in proliferation inhibition assay. Metformin can also augment apoptosis effect of these TKI-resistant cells to EGFR-TKIs. In retrospective cohort, a total of 85 patients were identified (cohort A), in which 28 patients had concurrent use of metformin. The objective response rate in metformin use group was significantly higher $(85.7 \%$ vs. $47.4 \%, \mathrm{P}=0.001)$. The median progression-free survival (PFS) and overall survival (OS) in metformin use group were significantly longer (21.6 vs. 9.2 months, $\mathrm{P}=0.000 ; 48.4$ vs. 36.6 months, $\mathrm{P}=0.049$ ). Further analysis revealed that metformin obviously prolonged the median PFS2 of osimertinib treatment among patients who progressed to prior line EGFR-TKIs due to secondary EGFR T790M mutation (cohort B).

Conclusions: Our study suggest that concurrent use of metformin could be beneficial to EGFR-mutant NSCLC patients treated with either first-line EGFR-TKIs or second-line osimertinib.

Keywords: Lung cancer; target therapy; EGFR-TKIs; metformin

Submitted Oct 30, 2020. Accepted for publication Jan 31, 2021.

doi: $10.21037 /$ tlcr-20-1153

View this article at: http://dx.doi.org/10.21037/tlcr-20-1153 


\section{Introduction}

Lung cancer remains the leading cause of cancer-related mortality around the world (1). Despite significant improvement in treatment of this malignancy, prognosis remains poor. In recent decades, non-small cell lung cancer (NSCLC) patients harboring sensitizing epidermal growth factor receptor $(E G F R)$ mutations, such as L858R point mutations (L858R) and exon 19 deletions (19DEL), have shown significant response to EGFR tyrosine kinase inhibitors (TKIs) (2). Several phase III randomized controlled clinical trials have confirmed the superior efficacy and safety of first-generation EGFR-TKIs such as gefitinib, icotinib, and erlotinib over chemotherapy, leading to them being recommended as the standard first-line treatment for advanced NSCLC patients with activating EGFR mutations (3-5). However, despite initial dramatic and rapid response to EGFR-TKIs, drug resistance is inevitable in most patients. The major mechanism for acquired resistance to EGFR-TKI is secondary T790M mutation, which accounts for approximately $50 \%$ (6). Various of studies have pursued approaches to delay or overcome resistance to first-generation TKIs, including exploring novel drug combinational strategies and developing nextgeneration TKIs. Third-generation EGFR-TKI, represented by osimertinib, exhibit powerful effects against tumors with the T790M resistance mutation $(7,8)$, but are ineluctably challenged by the issue of drug resistance as well (9-11). The clinical efficacy of osimertinib also varies even among patients with T790M mutations, and the objective response rate of osimertinib has remained at around $80 \%$ in first-line untreated NSCLC patients and around $60 \%$ in second-line treatment $(12,13)$. Thus, novel combinational strategies are urgently required to overcome therapeutic resistance to EGFR-TKIs in order to improve the clinical outcomes of NSCLC patients.

Studies have suggested that the prolonged use of metformin, an IGF-1R inhibitor, also an oral anti-diabetes agent commonly used in type 2 diabetes mellitus (T2DM) patients, is associated with survival benefits among NSCLC diabetic patients $(14,15)$. Preclinical researches had found that metformin surprisingly demonstrated anticancer effects through decreasing proliferation, promoting apoptosis and enhance autophagy of cancer cells (16). A preclinical study carried out by Li et al. showed that metformin could reverse EGFR-TKIs resistance through EMT reversal and inhibiting of IL-6 signaling pathway (17). A recent study also demonstrated that the combination of metformin and gefitinib could overcome primary resistance to gefitinib by targeting the IGF-1R signaling pathway (18). Several retrospective studies also indicated that metformin could improve survival in patients with lung cancer patients treated with EGFR-TKIs (19). However, the efficacy of concurrent use of metformin and EGFR-TKIs, especially third-generation TKIs, on EGFR-mutant NSCLC patients has not been well validated.

In our study, we carried out experiment to demonstrate the effects of metformin combining with gefitinib or osimertinib in resistant cell lines in aspect of proliferation and apoptosis. And then, we retrospectively reviewed the clinical efficacy of metformin with EGFR-TKIs in EGFRmutant NSCLC patients with diabetes. We stratified patients by metformin administration and compared the therapeutic responses and clinical outcomes in untreated NSCLC patients receiving first-generation EGFR-TKIs, as well as in patients receiving a second-line osimertinib treatment that acquired secondary T790M mutations.

We present the following article in accordance with STORBE reporting checklist. Available at http://dx.doi. org/10.21037/tlcr-20-1153.

\section{Methods}

\section{Cell culture and reagents}

Gefitinib and osimertinib were purchased from (Selleck, USA), both drugs were dissolved in dimethyl sulfoxide (DMSO) to obtain a stock solution of $10 \mathrm{mmol} / \mathrm{L}$ solution in $-20^{\circ} \mathrm{C}$. Metformin (Sigma-Aldrich, USA) was dissolved in deionized water and prepared for a $1 \mathrm{M}$ stock solution and stored at $-20^{\circ} \mathrm{C}$. Stock solutions were diluted to the appropriate concentrations with growth medium immediately before use.

Gefitinib-resistant human NSCLC cell line PC9R was provided by Tongji University (China). Following the protocol described in previous study, osimertinib-resistant cell line PC9R/OR was newly established by exposing PC9R to stepwise concentration of osimertinib (from 10 to $1.2 \mu \mathrm{M}$ in 9 days) and maintained in $1.2 \mu \mathrm{M}$ over 6 weeks. All cells were cultured at $37^{\circ} \mathrm{C}$ with $5 \% \mathrm{CO}_{2}$ in Dulbecco's Modified Eagle's Medium (DMEM) (Hyclon) that was supplemented with $10 \%$ fetal bovine serum (FBS) (Gibco), $100 \mu \mathrm{g} / \mathrm{mL}$ penicillin (Hyclon) and $100 \mu \mathrm{g} / \mathrm{mL}$ streptomycin (Hyclon).

\section{Growth inbibition assay}

Cells were seeded in 96-well cell plates at 2,000 cells/well in 
$100 \mu \mathrm{L}$ culture medium. After $12 \mathrm{~h}$ incubation, Cells were exposed to various treatment for $72 \mathrm{~h}$. The cell viability was assessed by using a Cell Counting Kit-8 (CCK8) (Wako). Optical density (OD) was measured at the wavelength of $570 \mathrm{~nm}$. Half maximal inhibitory concentration $\left(\mathrm{IC}_{50}\right)$ value was defined as the concentration of gefitinib or osimertinib needed for $50 \%$ reduction of growth. The combination indices $(\mathrm{CI})$ for drug interaction was calculated using $\mathrm{CI}=$ (D) $1 /(\mathrm{Dx}) 1+(\mathrm{D}) 2 /(\mathrm{Dx}) 2+\alpha(\mathrm{D}) 1(\mathrm{D}) 2 /(\mathrm{Dx}) 1(\mathrm{Dx}) 2$. Where (Dx) $1=\mathrm{IC}_{50}$ of drug 1 (EGFR-TKI) alone; (D) $1=\mathrm{IC}_{50}$ of drug 1 (TKI) in combination of drug 2 (metformin); $(\mathrm{Dx}) 2=\mathrm{IC}_{50}$ of drug 2 alone; (D) $2=\mathrm{IC}_{50}$ of drug 2 in combination of drug $1 ; \alpha=0$ for mutually exclusive or $\alpha=1$ for mutually nonexclusive modes of drug action.

\section{Detection of Apoptosis}

PC9R cells were treated with gefitinib $\left(\mathrm{IC}_{25}\right)$, metformin $(5 \mathrm{mM})$, or combination of gefitinib $\left(\mathrm{IC}_{25}\right)$ and metformin (5 mM), respectively; PC9R/OR cells were treated with osimertinib $\left(\mathrm{IC}_{25}\right)$, metformin $(5 \mathrm{mM})$, or combination of osimertinib $\left(\mathrm{IC}_{25}\right)$ and metformin $(5 \mathrm{mM})$, respectively. After $72 \mathrm{~h}$, cells were harvested and centrifugated for $5 \mathrm{~min}$ at $500 \times \mathrm{g}$, and then suspended at a density of $1 \times 10^{6}$ cells $/ \mathrm{mL}$. The Annexin V/PI apoptosis detection kit (BD, USA) was used to detect apoptosis according to the manufacturer's instructions. Data obtained were analyzed using FlowJo X 10.0. (BD, USA).

\section{Retrospective study population}

We screened 983 patients with advanced EGFR-mutant NSCLC in Shanghai Pulmonary Hospital between Jan 2015 to May 2018. A total of 85 patients with advanced EGFR-mutant NSCLC and type 2 diabetes mellitus (T2DM) who had received first-line EGFR-TKIs treatment were involved in the current study. Inclusion criteria were: histological or cytological confirmed NSCLCs harboring either L858R mutation or exon 19 deletion; receiving single EGFR-TKIs as first-line treatment; preexisting T2DM before the initiation of EGFR-TKI therapy; concurrent use of hypoglycemic drugs with EGFR-TKIs; and thorough documentation of response evaluation for patients.

\section{Clinical effects evaluation}

Clinicopathologic features including age, sex, Eastern Cooperative Oncology Group (ECOG) performance status
(PS), TNM stage, EGFR mutation status, type of antidiabetes medication, histological type, and smoking status were recorded. Smokers were defined as patients with a history of smoking $\geq 100$ cigarettes within their lifetime, whereas nonsmokers were defined as those with a history of smoking $<100$ cigarettes in their lifetime.

Tumor responses was recorded and evaluated every two to three months using computerized tomography (CT). According to the Response Evaluation Criteria in Solid Tumors version 1.1 (20), the response was classified as a complete response (CR), partial response (PR), progressive disease (PD), or stable disease (SD). The objective response rate $(\mathrm{ORR})$ was defined as follows: $\mathrm{ORR}=(\mathrm{CR}+\mathrm{PR}) /$ total cases $\times 100 \%$. The disease control rate (DCR) was calculated by follows: $(\mathrm{CR}+\mathrm{PR}+\mathrm{SD}) /$ total cases $\times 100 \%$. Progressionfree survival 1 (PFS1) was defined as the period from the date of initiating first-line EGFR-TKI treatment to the date of progression or of death. Overall survival 1 (OS1) was figured from initiating first-line EGFR-TKI treatment until any cause of death or, in surviving patients, the last follow-up. PFS2 was calculated from the date of secondline osimertinib treatment initiation to the date of disease progression or patient death. OS2 was calculated from the date of osimertinib initiation to the date of death from any cause or last follow-up.

\section{Statistical analysis}

Statistical analyses for in vitro experiments were performed using GraphPad Prism 7.0. The two-tailed $t$-test and ANOVA analysis were used to compare the continuous values. A $\mathrm{P}$ value $<0.05$ was considered to indicate significant difference.

All retrospective data analysis was performed using SPSS statistical software (version 22.0). Continuous variables were compared using $t$-test and ANOVA analysis, and categorical variables were compared across groups using the Chi-square test, Fisher exact test, Mann-Whitney U test, as appropriate. PFS and OS were analyzed using Kaplan-Meier curves, and differences between groups were compared using the log-rank test. Cox regression models were used to identify prognostic factors for survival. Results were considered statistically significant at when $\mathrm{P}$ value $<0.05$.

\section{Ethical statement}

The study was approved by the Ethics Committee of Shanghai Pulmonary Hospital (No. K18-089-1) and 
A

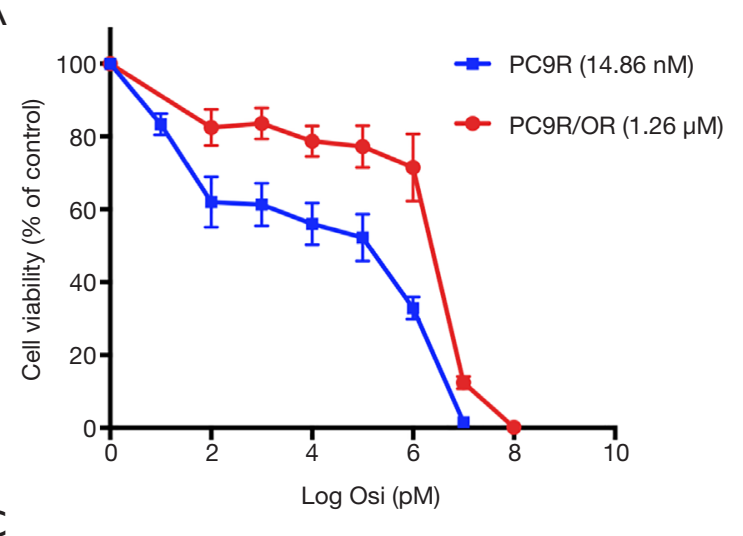

C

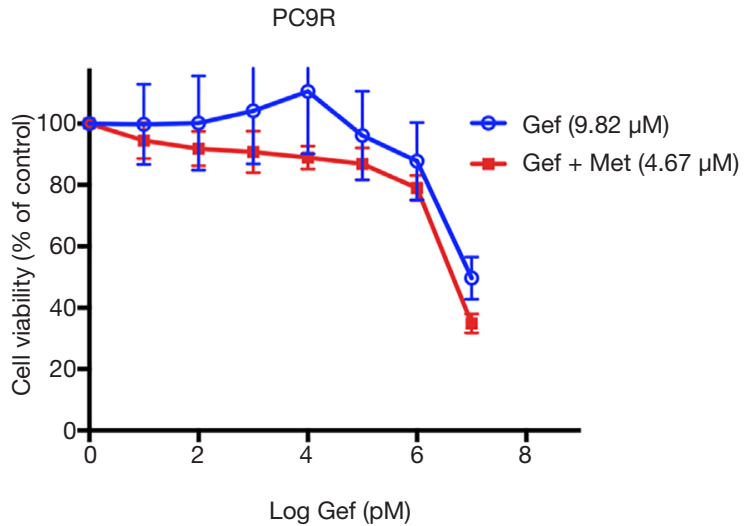

B

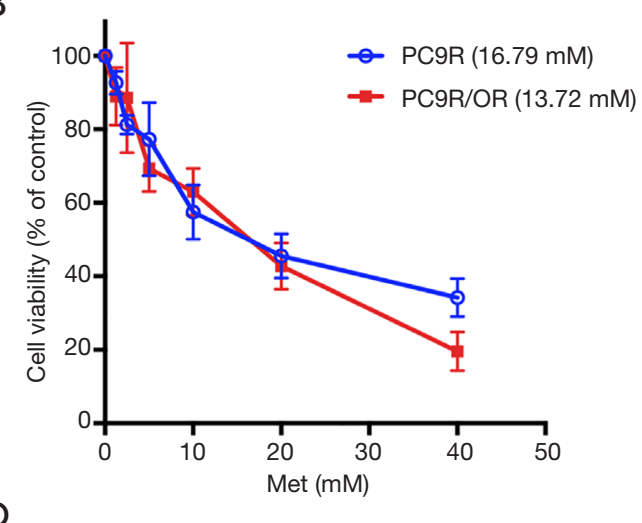

PC9R/OR

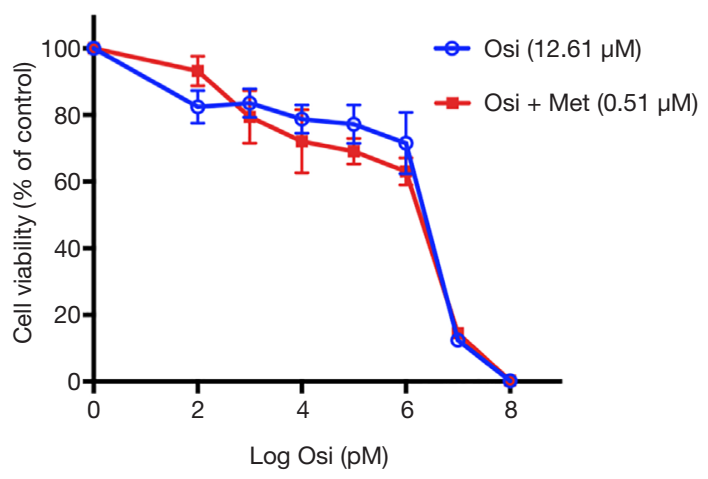

Figure 1 Concurrent use of metformin sensitized TKI-resistant cells to EGFR-TKIs. (A) PC9R and PC9R/OR cells were incubated with indicate concentration gradient of osimertinib for $72 \mathrm{~h}$. (B) PC9R and PC9R/OR cells were incubated with $5 \mathrm{mM}$ metformin alone for $72 \mathrm{~h}$. (C) PC9R cells were treated with indicate concentration gradient of gefitinib with or without $5 \mathrm{mM}$ metformin for $72 \mathrm{~h}$. (D) PC9R/OR cells were treated with indicate concentration gradient of osimertinib with or without $5 \mathrm{mM}$ metformin for $72 \mathrm{~h}$. Cell proliferation was measured by CCK8 assay. The bar graph reveals the $\mathrm{IC}_{50}$ values of TKI for the cells. Each data point represents the average value of six samples and is expressed as a percentage of the surviving cells relative to the untreated controls.

was performed according to the Declaration of Helsinki (as revised in 2013), and individual consent for this retrospective analysis was waived.

\section{Results}

\section{Establishment of osimertinib-resistant cells}

We successfully established osimertinib-resistant cell line PC9R/OR from gefitinib-resistant cell line PC9R. CCK8 assay showed the $\mathrm{IC}_{50}$ of PC9R and PC9R/OR to osimertinib were $14.86 \mathrm{nM}$ and $1.26 \mu \mathrm{M}$, respectively, the fold change of $\mathrm{IC}_{50}$ was 84.79 (Figure $1 A$ ). We also test their $\mathrm{IC}_{50}$ to metformin, were $16.79 \mathrm{mM}$ (PC9R) and $13.72 \mathrm{mM}$ (PC9R/OR) respectively (Figure $1 B$ ), and $5 \mathrm{mM}$ metformin slightly change cell viability in both cell lines.

\section{Metformin re-sensitized EGFR-TKI resistant NSCLC cells to EGFR-TKIs in vitro}

Cell proliferation assay demonstrated that PC9R cells were resistant to gefitinib, concurrent use of $5 \mathrm{mM}$ metformin re-sensitized PC9R to gefitinib (Figure 1C). Combination of gefitinib and metformin showed synergistic interaction (CI $=0.77$ ) with reduction in $\mathrm{IC}_{50}$ values of $9.82 \mu \mathrm{M}$ (gefitinib alone) to $4.67 \mu \mathrm{M}$ (with both agents) (Table 1). Meanwhile, metformin also sensitized PC9R/OR cells to osimertinib compared to osimertinib alone (Figure 1D). Combination of osimertinib and metformin showed synergistic interaction $(\mathrm{CI}=0.77)$ with reduction in $\mathrm{IC}_{50}$ values of $1.26 \mu \mathrm{M}$ 
Table 1 The $\mathrm{IC}_{50}$ values and CI for combination of EGRR-TKIs and metformin against TKI-resistant cell lines

\begin{tabular}{lcccccc}
\hline Cell line & TKI & TKI IC $_{50}$ & Metformin IC & TKI IC $_{50}$ in combination & Cl & Interpretation \\
\hline PC9R & Gefitinib & $9.82 \mu \mathrm{M}$ & $16.79 \mu \mathrm{M}$ & $4.67 \mu \mathrm{M}$ & 0.77 & Synergism \\
PC9R/OR & Osimertinib & $1.26 \mu \mathrm{M}$ & $13.72 \mu \mathrm{M}$ & $0.51 \mu \mathrm{M}$ & 0.77 & Synergism \\
\hline
\end{tabular}

$\mathrm{TKI}$, tyrosine kinase inhibitor; $\mathrm{Cl}$, combination indices.

A

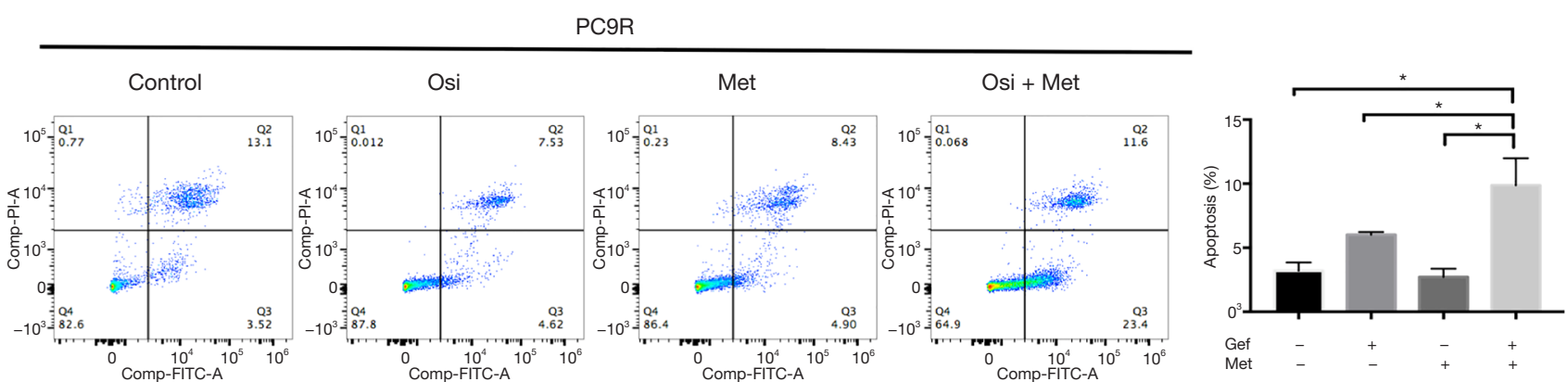

B

PC9R/OR
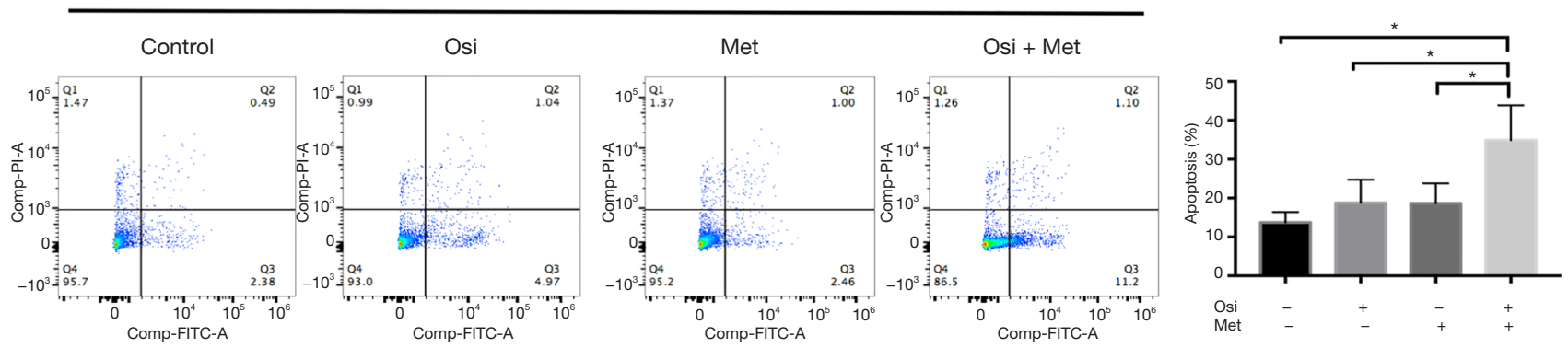

Figure 2 Concurrent use of metformin with EGFR-TKIs augment apoptosis in TKI-resistant cells. (A) The synergistic pro-apoptosis effects of gefitinib $\left(\mathrm{IC}_{25}\right)$ combined with metformin $(5 \mathrm{mM})$ on PC9R cells for $48 \mathrm{~h}$, as determined by Annexin V/PI staining assay and FACS analysis. (B) Osimertinib $\left(\mathrm{IC}_{25}\right)$ and metformin $(5 \mathrm{mM})$ synergistically enhanced the apoptosis of PC9R/OR cells after $48 \mathrm{~h}$ incubation, as assessed by Annexin V/PI staining assay. Statistical differences between groups were analyzed using one-way ANOVA ( ${ }^{*} \mathrm{P}<0.05$ compared with control).

(osimertinib alone) to $0.51 \mu \mathrm{M}$ (with both agents) (Table 1). These results suggest that metformin could sensitize TKIresistant cells to TKI treatment.

\section{EGFR-TKIs combined with metformin augments apoptosis in TKI-resistant NSCLC cell lines}

We also assessed whether metformin induces apoptosis in combination with TKIs in TKI-resistant cell lines. We treated PC9R cells with gefitinib $3.17 \mu \mathrm{M}\left(\mathrm{IC}_{25}\right)$ alone or in combination with metformin $(5 \mathrm{mM})$ for $48 \mathrm{~h}$ and then conducted Annexin V/PI staining followed by flow cytometry analysis. Result showed addition of metformin significantly increase apoptosis rate in PC9R cells $(\mathrm{P}<0.05)$
(Figure $2 A$ ). Meanwhile, combination use of osimertinib $420 \mathrm{nM}\left(\mathrm{IC}_{25}\right)$ and metformin $(5 \mathrm{mM})$ also significantly augment apoptosis in $\mathrm{PC} 9 \mathrm{R} / \mathrm{OR}$ cells $(\mathrm{P}<0.05)$ (Figure $2 B$ ). The results indicated that metformin can augment apoptosis effect of TKI-resistant cells to TKIs.

\section{Patient characteristics}

A total of 85 patients meet the inclusion criteria and were included in our study (cohort A), including 36 female patients and 49 male patients (Figure 3). The last followup time was Jan 2020. Clinical baseline characteristics are shown in Table 2. The median age was 65 (range, 36-84). Most patients were diagnosed with adenocarcinoma 


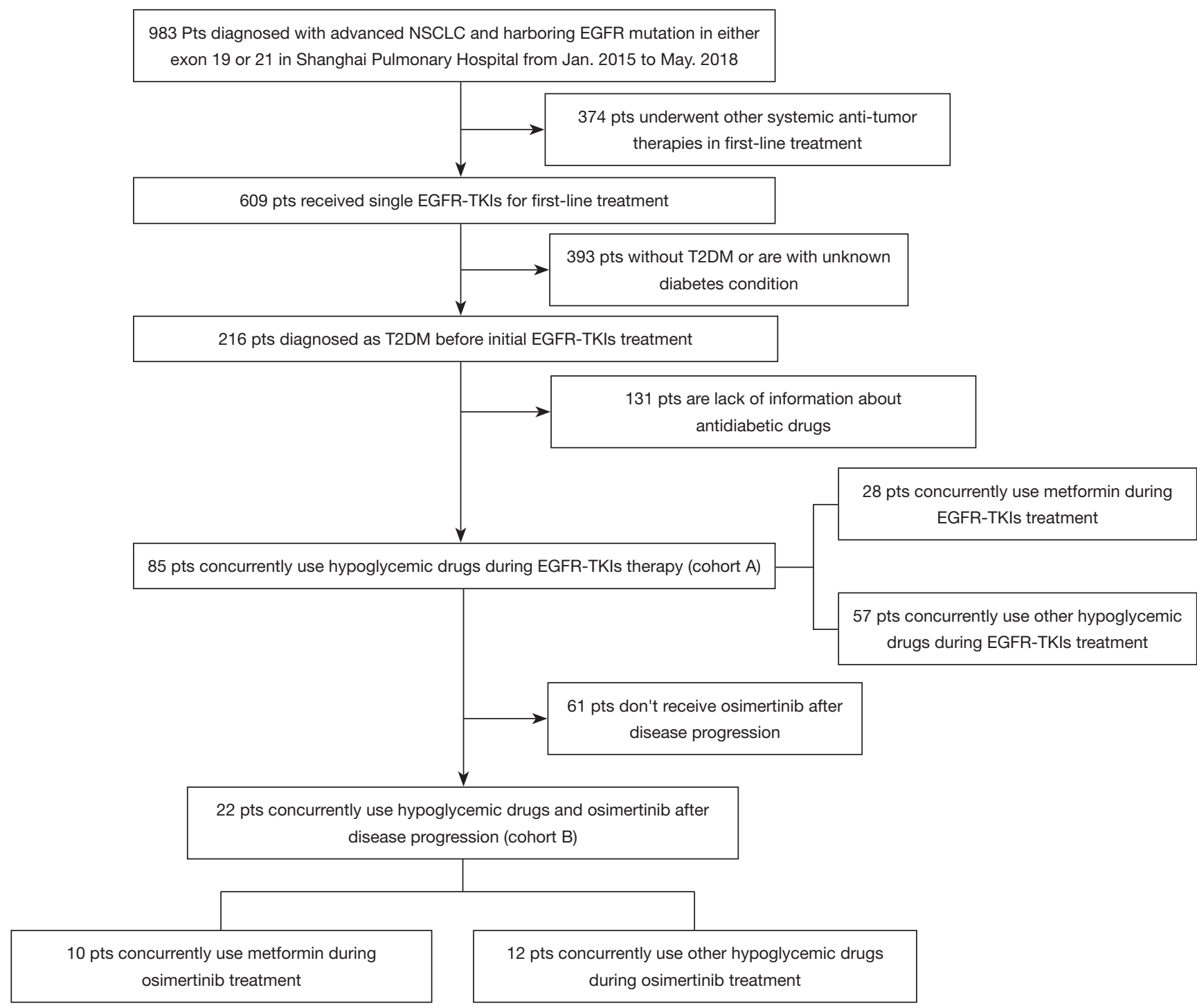

Figure 3 Flow diagram of patients studied.

(90.6\%, n=77) and were non-smokers (70.6\%, n=60). Only 10 patients $(11.8 \%)$ had an ECOG PS status $\geq 2$. Seven patients $(8.2 \%)$ were IB-IIIA at diagnosis, and 78 patients (91.8\%) were IIIB-IV. All patients were treated with firstline single-agent EGFR-TKIs (gefitinib: $n=53$, icotinib: $\mathrm{n}=21$, erlotinib: $\mathrm{n}=11$ ). All enrolled patients harbored sensitive EGFR mutations, of which 37 patients (43.5\%) had a 19DEL mutation and 48 patients (56.5\%) had a L858R mutation. The full cohort was divided into two groups based on metformin use; 28 patients were metformin users while 57 received other anti-diabetic medications such as insulin, acarbose, or sulfonylureas. All clinicopathologic features were well balanced between the two groups.

Among patients who developed acquired resistance to previous EGFR-TKIs, 22 were detected to have obtained a secondary EGFR T790M mutation and subsequently received single-agent osimertinib (cohort B). Clinical characteristics of this patient cohort are also shown in Table 2. Similarly, we dichotomized this cohort according to metformin exposure status. No significant differences in baseline clinical 
Table 2 Clinical characteristics of 85 patients with both EGFR-mutant advanced NSCLC and T2DM

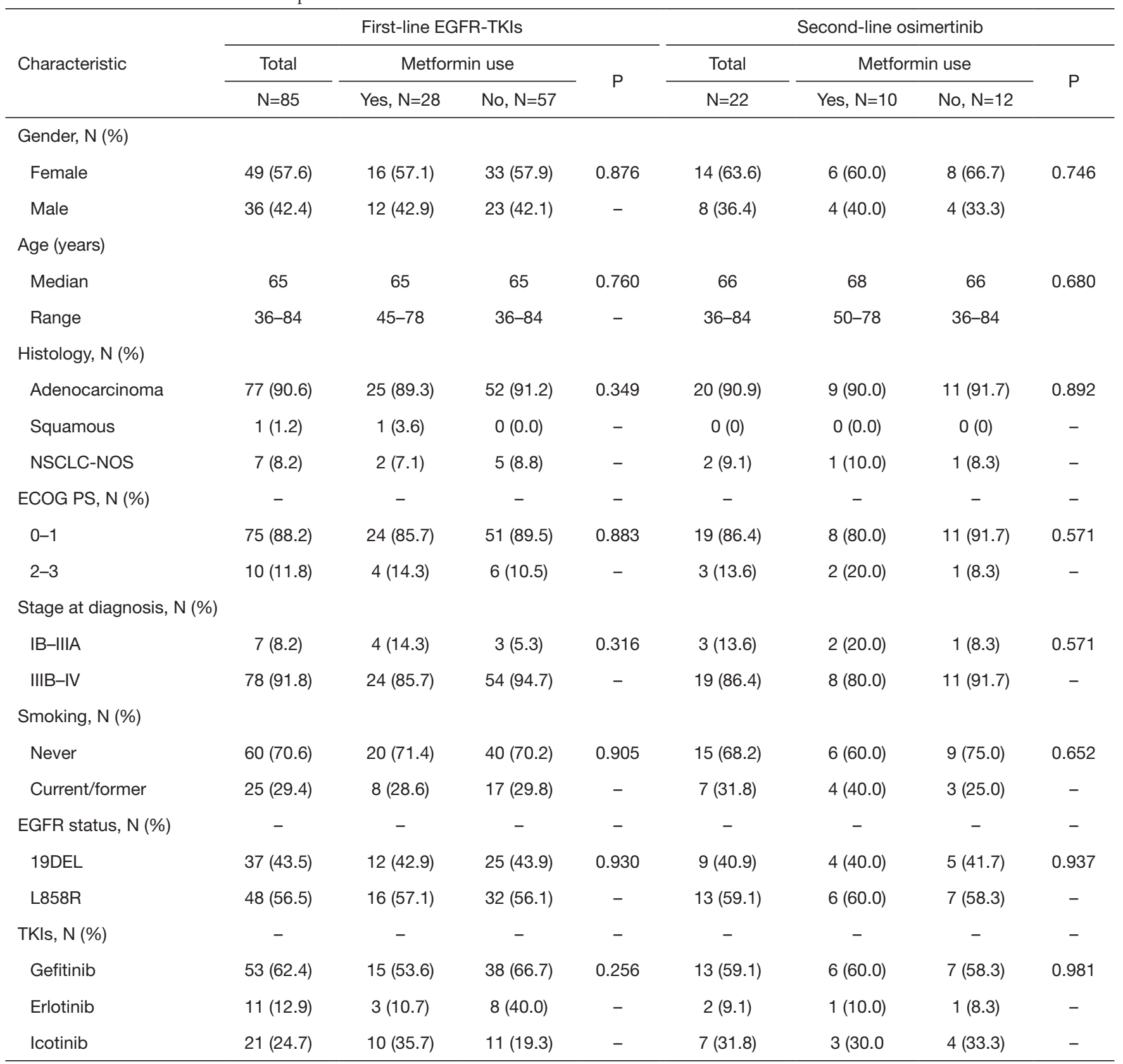

EGFR-TKI, epidermal growth factor receptor-tyrosine kinase inhibitor; NSCLC-NOS, non-small cell lung cancer-not otherwise specified; ECOG PS, Eastern Cooperative Oncology Group performance status.

characteristics were found between the groups.

\section{Concurrent use of metformin with EGFR-TKIs was associated with improved survival}

All 85 patients in full cohort were evaluable for best tumor response (Table 3). Of these, 51 patients displayed $\mathrm{PR}$ and 31 patients had SD, with another three patients that underwent PD. ORR and DCR were $60.0 \%$ and $96.5 \%$ respectively. A statistically significant inferior ORR was observed in patients with metformin use (ORR: $85.7 \%$ vs. $47.4 \%$, $\mathrm{P}=0.001)$. However, no difference was found between the 
Table 3 Response and disease control rates in EGFR-mutant NSCLC patients treated with EGFR-TKIs with or without metformin

\begin{tabular}{|c|c|c|c|c|c|c|c|c|}
\hline \multirow{2}{*}{ Response } & \multicolumn{4}{|c|}{ First-line EGFR-TKIs } & \multicolumn{4}{|c|}{ Second-line Osimertinib } \\
\hline & Total $\mathrm{N}=85$ & Yes & No & $\mathrm{P}$ & Total $\mathrm{N}=22$ & Yes & No & $\mathrm{P}$ \\
\hline $\mathrm{CR}$ & - & - & - & & - & - & - & \\
\hline PR & 51 & 24 & 27 & & 10 & 6 & 4 & \\
\hline PD & 3 & - & 3 & & 2 & - & 2 & \\
\hline ORR & $60.0 \%$ & $85.7 \%$ & $47.4 \%$ & 0.001 & $45.5 \%$ & $60.0 \%$ & $33.3 \%$ & 0.412 \\
\hline DCR & $96.5 \%$ & $100.0 \%$ & $94.7 \%$ & 0.548 & $90.9 \%$ & $100.0 \%$ & $83.3 \%$ & 0.481 \\
\hline
\end{tabular}

EGFR-TKI, epidermal growth factor receptor-tyrosine kinase inhibitor; CR, complete response; PR, partial response; SD, stable disease; $\mathrm{PD}$, progressive disease; progression-free survival; ORR, objective response rate; DCR, disease control rate.

two groups in terms of DCR (100.0\% vs. $94.7 \%, \mathrm{P}=0.548)$.

Next, we analyzed survival benefit in response to first-line EGFR-TKIs treatment (Table 4). The overall median PFS1 and OS1 values were 12.2 months (95\% CI: 10.2-14.2 months) and 42.3 months (95\% CI: 30.8-53.8), respectively. Patients in the metformin group showed a significantly longer PFS1 than patients who were not exposed to metformin (median PFS1, 21.6 vs. 9.2 months, $\mathrm{P}=0.000$ ) (Figure 4A). The metformin group also demonstrated superior overall survival (median OS1, 48.4 vs. 36.6 months, $\mathrm{P}=0.049$ ) (Figure 4B).

The 22 patients who developed resistance to first-line EGFR-TKIs and had confirmed T790M mutations were evaluable for best tumor response to second-line osimertinib treatment (Table 3). Of these, 10 patients had PR, 10 patients had SD, and 2 patients displayed progression. Across this patient cohort, the objective response rate and DCR were $45.5 \%$ and $90.9 \%$, respectively. The ORR and DCR values trended higher in patients with metformin use (ORR: $60.0 \%$ vs. $33.3 \%$, DCR: $100.0 \%$ vs. $83.3 \%$ ), but no statistical significance was observed between the two groups.

Survival benefit in response to second-line osimertinib treatment was also evaluated (Table 4). The median PFS2 with second-line osimertinib was 14.5 months (95\% CI: 9.1-19.9 months). Significantly longer PFS2 was seen in the metformin group (median PFS2, 20.5 vs. 11.3 months, $\mathrm{P}=0.002$ ) (Figure 4C). No difference was observed in OS2 (median OS2, 28.4 vs. 27.1 months, $\mathrm{P}=0.829$ ) (Figure 4D). The impact of metformin use on PFS1 and OS1 within patient subgroups was further evaluated (Figure 5). For patients who received first-line EGFR-TKIs treatment, metformin use had a consistently positive effect on the survival benefit.

\section{Other clinical variables were not associated with the differential benefit of survival}

To further evaluate the potential impact of clinical variables on the therapeutic efficacy of treatment with first-line EGFR-TKIs and second-line osimertinib, we performed multivariate analysis on patient cohort A and cohort B separately (Table 5). Typical factors of sex, age, ECOG PS, histology, smoking history, EGFR driver mutation type, and metformin use status were included in the Cox regression model. Patients in the metformin use group displayed a remarkably better PFS1 and also PFS2 compared with those not using metformin (HR $=0.177,95 \%$ CI: 0.098-0.318, $\mathrm{P}=0.000 ; \mathrm{HR}=0.348,95 \% \mathrm{CI}: 0.135-0.897, \mathrm{P}=0.029)$. The patients using metformin also had significant benefit to OS1 (HR $=0.112$, 95\% CI: 0.023-0.549, P=0.007). However other clinical characteristics were not prognostic factor neither in PFS nor in OS.

\section{Discussion}

In EGFR-mutant NSCLC patients, blockade of oncogenic EGFR signaling by EGFR-TKIs results in dramatic clinical response. However, almost all patients with EGFR-mutant lung tumors treated with EGFR-TKIs will eventually progress (21). The underlying mechanisms associated with resistance to first-generation EGFR-TKIs have been well discussed. In addition to the major mechanisms of secondary T790M mutations in EGFR and amplification of the $M E T$ oncogene, which account for approximately $50 \%$ 
of acquired resistance to TKIs (22), various other molecular mechanisms are also involved, including hepatocyte growth factor (HGF) overexpression, epithelial to mesenchymal transition (EMT), and activation of pathways such as the insulin-like growth factor-1 receptor (IGF-1R) pathway (23-25). Besides, 20-30\% patients exhibit primary resistance to EGFR-TKIs, variations in IGF-1R, BIM, and T790M are the common causes (26). Strategies to overcome drug resistance were also divided to several directions including adopting third-generation EGFR-TKI, in combination with EGFR-targeting antibody or bypass signaling pathway inhibitors, and switching platinum-based therapy, respectively (26).

Although the third-generation EGFR-TKI osimertinib shows superior efficacy in patients with a secondary EGFR T790M mutation that confers resistance to prior EGFRTKIs, the survival of these patients is also heterogeneous and drug resistance is also inevitable $(8,27)$. So far, resistance mechanisms to osimertinib can be roughly divided into two categories based on whether they are related to EGFR pathway (9). Major EGFR-dependent mechanisms including EGFR C797S mutation, EGFR amplification, and EGFR T790M loss, besides, EGFRindependent mechanisms mainly contain activation of alternative pathway, SCLC transformation, and epithelial mesenchymal transition (EMT) (28). The principle to overcome osimertinib resistance is quite the same as previous EGFR-TKIs. These facts motivated us to explore novel treatment strategies.

Metformin is a widely used anti-diabetic drug, also an IGF-1R inhibitor, which surprisingly exerts anticancer actions. The anti-tumor effects of metformin were initially observed in epidemiological studies, which demonstrated that metformin could decrease cancer incidence and mortality in diabetes $(29,30)$. Numerous of preclinical studies had explored the potential anti-tumor mechanisms of metformin in lung, liver, endometrial, esophagus, melanoma, and endometrial cancers cell lines (16). Generally, metformin might impede tumor proliferation, promote apoptosis through modulating cancer cell signaling and metabolism (31). However, the concentration of metformin as monotherapy in cancer treatment in vitro were supra-physiological that could not be applied in clinical (32). In contrast, lower dose of metformin used as adjuvant treatment with other anti-cancer therapies were tolerable and demonstrated promising efficacy (16).

Previous preclinical have demonstrated that metformin showed significant growth inhibitory ability and pro- 
A

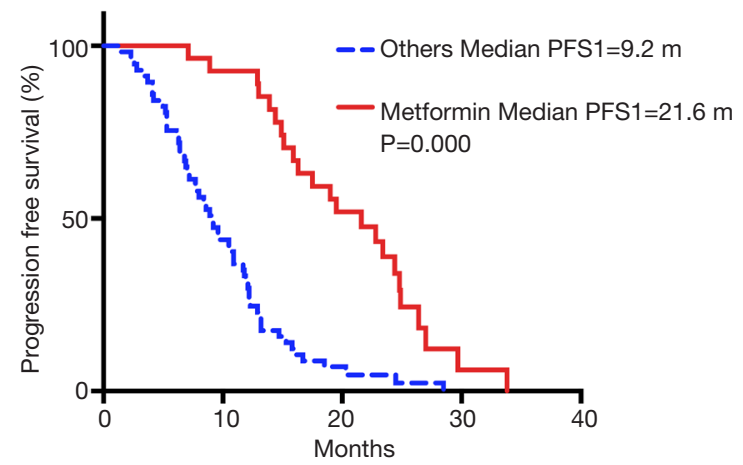

C

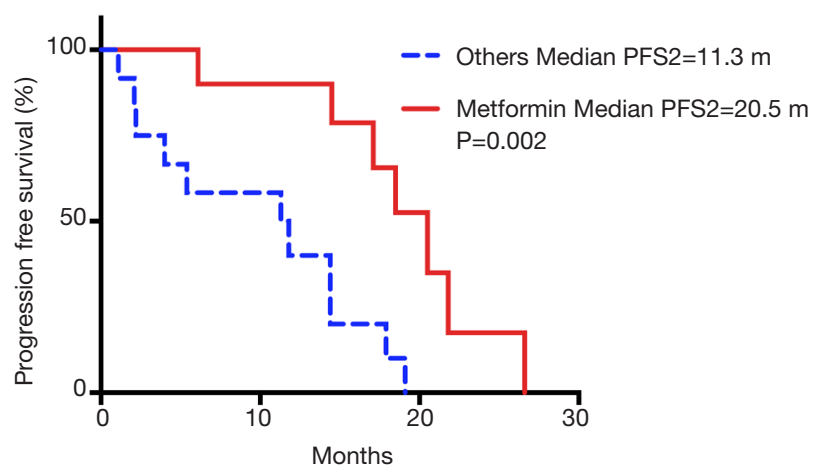

B

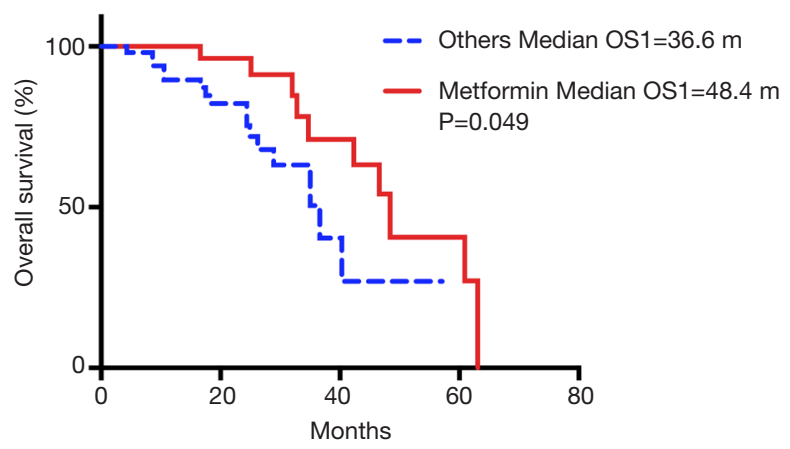

D

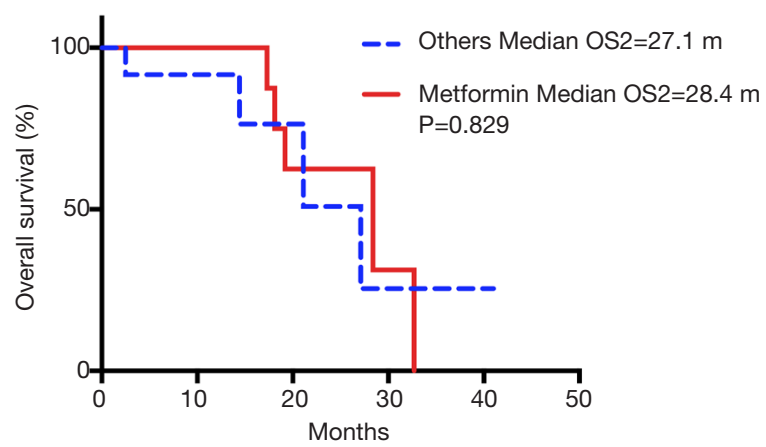

Figure 4 Concurrent use of metformin improved survival of EGFR-mutant patients with advanced NSCLC and T2DM. (A) PFS1 stratified by metformin use in first-line EGFR-TKIs treatment. (B) OS1 stratified by metformin use in first-line EGFR-TKIs treatment. (C) PFS2 stratified by metformin use in second-line osimertinib treatment. (D) OS2 stratified by metformin use in second-line osimertinib treatment. Full line indicates patients who use metformin. Dotted line indicates patients who use hypoglycemic drugs other than metformin.

apoptotic effects on tumor cells in many cancer models including lung cancer (33-35). Previous study has demonstrated that the combination of metformin and gefitinib induced a synergistic effect in NSCLC cell lines by modulating LKB1-AMPK-mTOR pathway (36). In another study, $\mathrm{Li}$ et al. showed that metformin effectively restored the sensitivity of TKI-resistant lung cancer cells to erlotinib or gefitinib by reversing EMT and suppressing IL6/STAT3 signaling activation (17). The combination of metformin and gefitinib has also been demonstrated to overcome primary resistance to EGFR-TKIs in NSCLC cell lines via targeting the IGF-1R signaling pathway (18). Recently, Hayakawa et al. showed IGF-1R activation might confer one of mechanisms of acquired osimertinib resistance in established osimertinib-resistant cells, additional treatment of IGF-1R inhibitor might overcome this resistance (37). We further validate these findings in gefitinib-resistant cells and osimertinib-resistant cells in vitro. Concurrent use of metformin could sensitize TKI-resistant cells to TKI treatment with CI value of 0.77 , which means the effect of two agents are slightly synergism. In apoptosis analysis, low dosage of EGFR-TKI $\left(\mathrm{IC}_{25}\right)$ and metformin $(5 \mathrm{mM})$ alone didn't increase apoptosis in both TKI-resistant cells, whereas combination of EGFR-TKIs and metformin demonstrated significant augment of apoptosis. These experiments showed metformin did have synergy effect with EGFR-TKI in aspect of cell proliferation inhibition and pro-apoptosis in TKI-resistant cells.

Based on preclinical studies, we believe that the use of metformin may overcome EGFR-TKI resistance and have a promising effect in improving outcomes of NSCLC patients. Related retrospective clinical studies are still needed to evaluate the clinical efficacy of this combination in NSCLC patients with EGFR-activating mutations. 

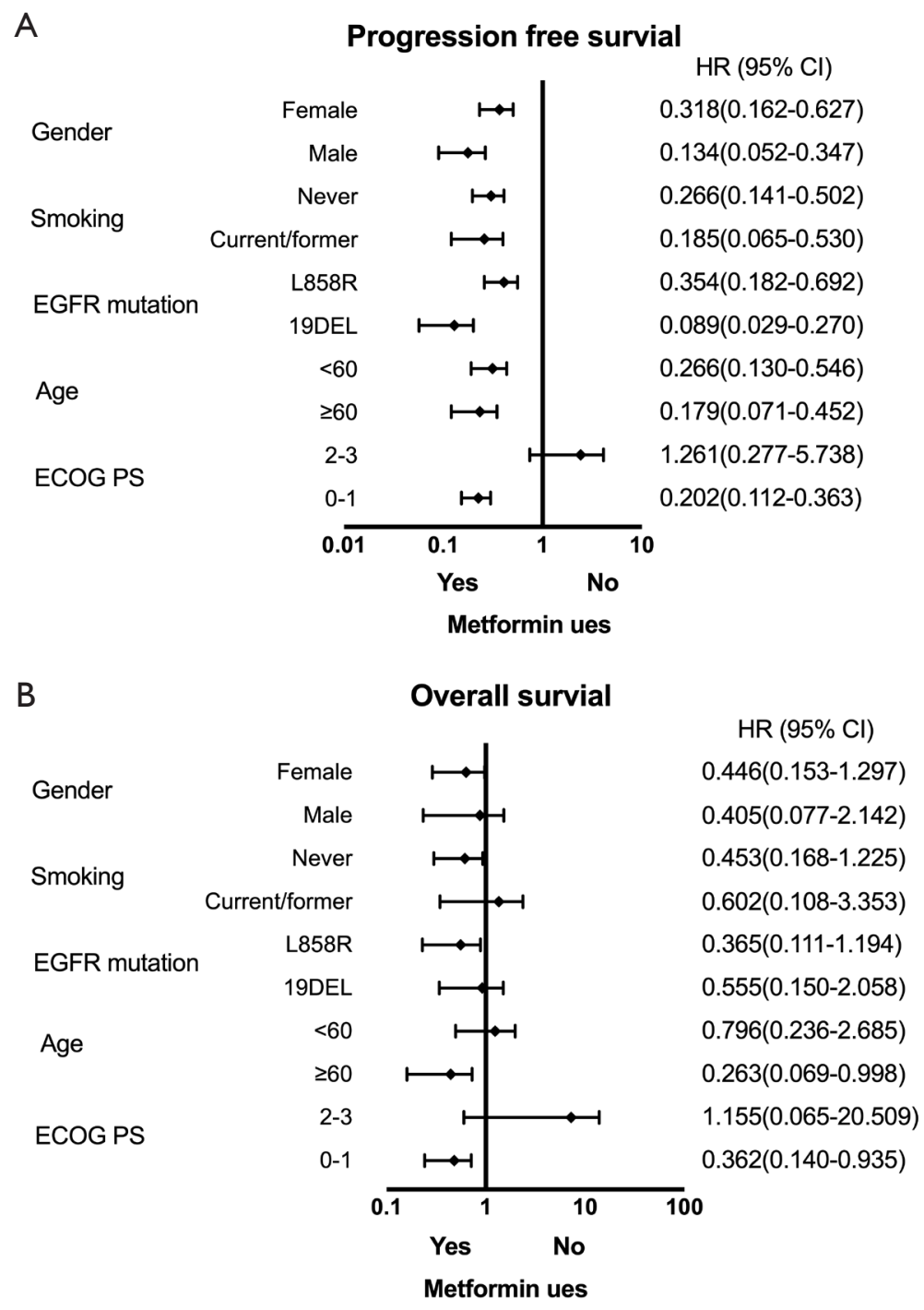

Figure 5 Forest plot of subgroup analysis by baseline characteristics for PFS1 (A) and OS1 (B) in patients treated with first-line EGFR-TKI and metformin.

It has been reported that metformin use is related to prolonged survival of lung cancer patients with diabetes in retrospective studies $(14,15,38,39)$. Levy and Doyen also reviewed concurrent metformin used as an anticancer agent in NSCLC patients and its possible molecular actions (19). In all, most of these retrospective researches showed that metformin could improve survival in patients with lung cancer patients treated with EGFR-TKIs.

Few randomized and prospective trails were carried out to evaluate efficacy of EGFR-TKI in combination with metformin in NSCLC patients with EGFR mutations. A double blinded, randomized phase II trials reported that no significant differences were observed in 1-year PFS, OS, and ORR between gefitinib plus placebo group versus gefitinib plus metformin group (40). In contrast, another randomized phase II trial demonstrated that addition of metformin to EGFR-TKIs (gefitinib, erlotinib and afatinib) significantly prolonged median PFS and OS compared to EGFR-TKI monotherapy in patients with EGFR mutated advanced adenocarcinoma (41).

In our retrospective study, we found that in EGFRmutant NSCLC patients treated with first-line gefitinib, 
Table 5 Multivariate analysis of clinical factors for patients in the cohort

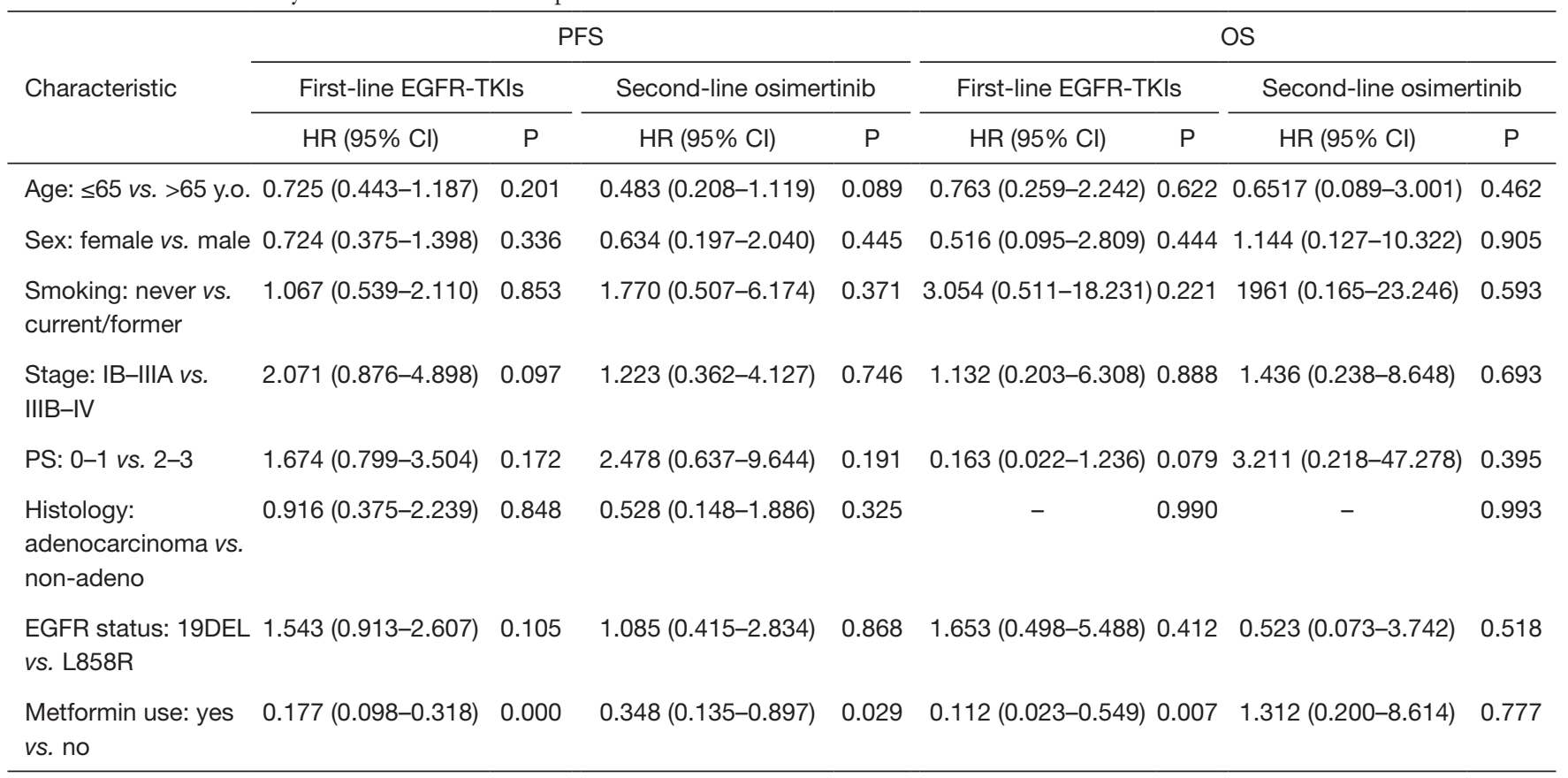

EGFR-TKI, epidermal growth factor receptor-tyrosine kinase inhibitor; HR, hazard ratio; Cl, confidence interval; y.o., years old; PS, performance status.

erlotinib, or icotinib, concurrent metformin use was related to higher ORR. Although the DCRs of the metformin and non-metformin groups were quite similar, we noticed that all of the three primary progressed patients belonged to the non-metformin group. Patients who received firstgeneration EGFR-TKIs plus metformin significantly improved PFS and OS over patients received other antidiabetes medications. Our results were consistent with a previous study carried out by Chen et al. that showed metformin seemed to have a synergistic effect with EGFR-TKIs in the treatment of T2DM NSCLC patients harboring EGFR mutations (39), which further confirms the clinical benefits of metformin in NSCLC patients treated with first-line EGFR-TKIs.

More noteworthy is our analysis with sub-cohort patients having a secondary EGFR T790M mutation that conferred resistance to prior EGFR-TKIs. Osimertinib is a multi-selective third-generation irreversible inhibitor of both EGFR sensitizing and T790M resistance mutations that spares wild-type EGFR (7). While the initial response to osimertinib is dramatic, the emergence of resistance is still inevitable (42). Therefore, we further compared the efficiency between use of osimertinib with or without metformin. In patients who developed acquired resistance to first-line EGFR-TKIs through T790M mutation, we found that metformin uses obviously improved the median PFS of osimertinib. The metformin group also showed higher ORR and DCR, though no statistical significance was observed. One possible reason for not achieving significance is the limited sample size of this patient cohort. In addition, no significant differences were observed in median OS between patients treated with metformin and patients treated with other hypoglycemic agents rather than metformin. One explanation for this result is the relatively short period of observation. If continue to follow-up, significances in OS may appear.

Since our study focused on NSCLC patients with T2DM, it remains unclear whether addition of metformin is tolerable in patients without diabetes. The phase II trials carried out by Arrieta $e t a l$. had evaluated the efficacy and safety of standard dose of metformin $(500 \mathrm{mg}$, twice a day) in combination of EGFR-TKI therapy versus standard EGFR-TKI monotherapy in EGFR-mutant NSCLC. It reported that the adverse effects were tolerable and comparable in both arms, and the most common Grade 3 or 4 adverse effects in both treatment groups were diarrhea, rash, nausea and mucositis (41). Metformin not only displayed the synergistic effect in anti-tumor with EGFR-TKI with 
a satisfied safe profile, it was also accessible and affordable. Taken together, our findings may provide important reference for clinicians supporting the use of metformin in advanced EGFR-mutant NSCLC patients treatment.

The limitations of the current study must be acknowledged. First, as a retrospective study preformed in a single institution, selection bias was inevitable. Second, the relatively small size of the patient cohort, especially patients receiving osimertinib, may limit our ability to adjust for confounding factors. Third, as this study focused on patients with T2DM who took metformin, we were unable to assess the influence of metformin in nondiabetic patients. Further prospective studies with a larger patient cohort are necessary to confirm our findings.

\section{Conclusions}

Concurrent use of metformin can improve patient response and delay resistance to NSCLC patients treated with either first-line EGFR-TKIs or second-line osimertinib.

\section{Acknowledgments}

The abstract of this work has been accepted for poster presentation at the IASLC 2020 World Conference on Lung Cancer.

Funding: This work was supported by grants from the National Natural Science Foundation of China [NO. 81871865], Shanghai Science and Technology Committee Foundation (NO. 19411950300), and Shanghai Municipal Health Commission (2014 Shanghai Leading Talent Project to CZ).

\section{Footnote}

Reporting Checklist: The authors have completed the STROBE reporting checklist. Available at http://dx.doi. org/10.21037/tlcr-20-1153

Data Sharing Statement: Available at http://dx.doi. org/10.21037/tlcr-20-1153

Conflicts of Interest: All authors have completed the ICMJE uniform disclosure form (available at http://dx.doi. org/10.21037/tlcr-20-1153). CZ serves as an unpaid editorial board member of Translational Lung Cancer Research from Aug 2020 to Jul 2022. The other authors have no conflicts of interest to declare.
Ethical Statement: The authors are accountable for all aspects of the work in ensuring that questions related to the accuracy or integrity of any part of the work are appropriately investigated and resolved. The study was conducted in accordance with the Declaration of Helsinki (as revised in 2013). The study was approved by ethics board of Shanghai Pulmonary Hospital (No. K18-089-1) and individual consent for this retrospective analysis was waived.

Open Access Statement: This is an Open Access article distributed in accordance with the Creative Commons Attribution-NonCommercial-NoDerivs 4.0 International License (CC BY-NC-ND 4.0), which permits the noncommercial replication and distribution of the article with the strict proviso that no changes or edits are made and the original work is properly cited (including links to both the formal publication through the relevant DOI and the license). See: https://creativecommons.org/licenses/by-nc-nd/4.0/.

\section{References}

1. Torre LA, Bray F, Siegel RL, et al. Global cancer statistics, 2012. CA Cancer J Clin 2015;65:87-108.

2. Castellanos E, Feld E, Horn L. Driven by Mutations: The Predictive Value of Mutation Subtype in EGFRMutated Non-Small Cell Lung Cancer. J Thorac Oncol 2017;12:612-23.

3. Mitsudomi T, Morita S, Yatabe Y, et al. Gefitinib versus cisplatin plus docetaxel in patients with non-small-cell lung cancer harbouring mutations of the epidermal growth factor receptor (WJTOG3405): an open label, randomised phase 3 trial. Lancet Oncol 2010;11:121-8.

4. Shi YK, Wang L, Han BH, et al. First-line icotinib versus cisplatin/pemetrexed plus pemetrexed maintenance therapy for patients with advanced EGFR mutation-positive lung adenocarcinoma (CONVINCE): a phase 3, open-label, randomized study. Ann Oncol 2017;28:2443-50.

5. Zhou C, Wu YL, Chen G, et al. Erlotinib versus chemotherapy as first-line treatment for patients with advanced EGFR mutation-positive non-small-cell lung cancer (OPTIMAL, CTONG-0802): a multicentre, open-label, randomised, phase 3 study. Lancet Oncol 2011;12:735-42.

6. Papadimitrakopoulou VA, Mok TS, Han JY, et al. Osimertinib versus platinum-pemetrexed for patients with EGFR T790M advanced NSCLC and progression on a prior EGFR-tyrosine kinase inhibitor: AURA3 overall survival analysis. Ann Oncol 2020;31:1536-44. 
7. Cross DA, Ashton SE, Ghiorghiu S, et al. AZD9291, an irreversible EGFR TKI, overcomes T790M-mediated resistance to EGFR inhibitors in lung cancer. Cancer Discov 2014;4:1046-61.

8. Mok TS, Wu YL, Ahn MJ, et al. Osimertinib or PlatinumPemetrexed in EGFR T790M-Positive Lung Cancer. N Engl J Med 2017;376:629-40.

9. Piotrowska Z, Isozaki H, Lennerz JK, et al. Landscape of Acquired Resistance to Osimertinib in EGFR-Mutant NSCLC and Clinical Validation of Combined EGFR and RET Inhibition with Osimertinib and BLU-667 for Acquired RET Fusion. Cancer Discov 2018;8:1529-39.

10. Yang Z, Yang N, Ou Q, et al. Investigating Novel Resistance Mechanisms to Third-Generation EGFR Tyrosine Kinase Inhibitor Osimertinib in Non-Small Cell Lung Cancer Patients. Clin Cancer Res 2018;24:3097-107.

11. Wang S, Tsui ST, Liu C, et al. EGFR C797S mutation mediates resistance to third-generation inhibitors in T790M-positive non-small cell lung cancer. J Hematol Oncol 2016;9:59.

12. Yang JC, Ahn MJ, Kim DW, et al. Osimertinib in Pretreated T790M-Positive Advanced Non-SmallCell Lung Cancer: AURA Study Phase II Extension Component. J Clin Oncol 2017;35:1288-96.

13. Soria JC, Ohe Y, Vansteenkiste J, et al. Osimertinib in Untreated EGFR-Mutated Advanced Non-Small-Cell Lung Cancer. N Engl J Med 2018;378:113-25.

14. Lin J, Gill A, Zahm SH, et al. Metformin use and survival after non-small cell lung cancer: A cohort study in the US Military health system. Int J Cancer 2017;141:254-63.

15. Arrieta O, Varela-Santoyo E, Soto-Perez-de-Celis E, et al. Metformin use and its effect on survival in diabetic patients with advanced non-small cell lung cancer. BMC Cancer 2016;16:633.

16. Morales DR, Morris AD. Metformin in cancer treatment and prevention. Annu Rev Med 2015;66:17-29.

17. Li L, Han R, Xiao H, et al. Metformin sensitizes EGFRTKI-resistant human lung cancer cells in vitro and in vivo through inhibition of IL-6 signaling and EMT reversal. Clin Cancer Res 2014;20:2714-26.

18. Pan YH, Jiao L, Lin CY, et al. Combined treatment with metformin and gefitinib overcomes primary resistance to EGFR-TKIs with EGFR mutation via targeting IGF-1R signaling pathway. Biologics 2018;12:75-86.

19. Levy A, Doyen J. Metformin for non-small cell lung cancer patients: Opportunities and pitfalls. Crit Rev Oncol Hematol 2018;125:41-7.

20. Eisenhauer EA, Therasse P, Bogaerts J, et al. New response evaluation criteria in solid tumours: revised RECIST guideline (version 1.1). Eur J Cancer 2009;45:228-47.

21. Lee DH. Treatments for EGFR-mutant non-small cell lung cancer (NSCLC): The road to a success, paved with failures. Pharmacol Ther 2017;174:1-21.

22. Remon J, Moran T, Majem M, et al. Acquired resistance to epidermal growth factor receptor tyrosine kinase inhibitors in EGFR-mutant non-small cell lung cancer: a new era begins. Cancer Treat Rev 2014;40:93-101.

23. Yano S, Wang W, Li Q, et al. Hepatocyte growth factor induces gefitinib resistance of lung adenocarcinoma with epidermal growth factor receptor-activating mutations. Cancer Res 2008;68:9479-87.

24. Yauch RL, Januario T, Eberhard DA, et al. Epithelial versus mesenchymal phenotype determines in vitro sensitivity and predicts clinical activity of erlotinib in lung cancer patients. Clin Cancer Res 2005;11:8686-98.

25. Guix M, Faber AC, Wang SE, et al. Acquired resistance to EGFR tyrosine kinase inhibitors in cancer cells is mediated by loss of IGF-binding proteins. J Clin Invest 2008;118:2609-19.

26. Tan CS, Gilligan D, Pacey S. Treatment approaches for EGFR-inhibitor-resistant patients with non-small-cell lung cancer. Lancet Oncol 2015;16:e447-e59.

27. Liao BC, Lin CC, Lee JH, et al. Optimal management of EGFR-mutant non-small cell lung cancer with disease progression on first-line tyrosine kinase inhibitor therapy. Lung Cancer 2017;110:7-13.

28. Tang $\mathrm{ZH}, \mathrm{Lu}$ JJ. Osimertinib resistance in non-small cell lung cancer: Mechanisms and therapeutic strategies. Cancer Lett 2018;420:242-6.

29. Evans JM, Donnelly LA, Emslie-Smith AM, et al. Metformin and reduced risk of cancer in diabetic patients. BMJ 2005;330:1304-5.

30. Heckman-Stoddard BM, DeCensi A, Sahasrabuddhe VV, et al. Repurposing metformin for the prevention of cancer and cancer recurrence. Diabetologia 2017;60:1639-47.

31. Pernicova I, Korbonits M. Metformin--mode of action and clinical implications for diabetes and cancer. Nat Rev Endocrinol 2014;10:143-56.

32. Pollak MN. Investigating metformin for cancer prevention and treatment: the end of the beginning. Cancer Discov 2012;2:778-90.

33. Cho SW, Yi KH, Han SK, et al. Therapeutic potential of metformin in papillary thyroid cancer in vitro and in vivo. Mol Cell Endocrinol 2014;393:24-9.

34. Salani B, Maffioli S, Hamoudane M, et al. Caveolin-1 is essential for metformin inhibitory effect on IGF1 action in 
non-small-cell lung cancer cells. FASEB J 2012;26:788-98.

35. Lengyel E, Litchfield LM, Mitra AK, et al. Metformin inhibits ovarian cancer growth and increases sensitivity to paclitaxel in mouse models. Am J Obstet Gynecol 2015;212:479.e1-10.

36. Morgillo F, Sasso FC, Della Corte CM, et al. Synergistic effects of metformin treatment in combination with gefitinib, a selective EGFR tyrosine kinase inhibitor, in LKB1 wild-type NSCLC cell lines. Clin Cancer Res 2013;19:3508-19.

37. Hayakawa D, Takahashi F, Mitsuishi Y, et al. Activation of insulin-like growth factor-1 receptor confers acquired resistance to osimertinib in non-small cell lung cancer with EGFR T790M mutation. Thorac Cancer 2020;11:140-9.

38. Tian RH, Zhang YG, Wu Z, et al. Effects of metformin on survival outcomes of lung cancer patients with type 2 diabetes mellitus: a meta-analysis. Clin Transl Oncol 2016;18:641-9.

39. Chen H, Yao W, Chu Q, et al. Synergistic effects of

Cite this article as: Han R, Jia Y, Li X, Zhao C, Zhao S, Liu S, Liu Y, Chen D, Zhang Q, Liu X, Shi J, Li J, Zhou C. Concurrent use of metformin enhances the efficacy of EGFRTKIs in patients with advanced EGFR-mutant non-small cell lung cancer-an option for overcoming EGFR-TKI resistance. Transl Lung Cancer Res 2021;10(3):1277-1291. doi: 10.21037/ tlcr-20-1153 metformin in combination with EGFR-TKI in the treatment of patients with advanced non-small cell lung cancer and type 2 diabetes. Cancer Lett 2015;369:97-102.

40. Li L, Jiang L, Wang Y, et al. Combination of Metformin and Gefitinib as First-Line Therapy for Nondiabetic Advanced NSCLC Patients with EGFR Mutations: A Randomized, Double-Blind Phase II Trial. Clin Cancer Res 2019;25:6967-75.

41. Arrieta O, Barron F, Padilla MS, et al. Effect of Metformin Plus Tyrosine Kinase Inhibitors Compared With Tyrosine Kinase Inhibitors Alone in Patients With Epidermal Growth Factor Receptor-Mutated Lung Adenocarcinoma: A Phase 2 Randomized Clinical Trial. JAMA Oncol 2019;5:e192553.

42. Lim SM, Syn NL, Cho BC, et al. Acquired resistance to EGFR targeted therapy in non-small cell lung cancer: Mechanisms and therapeutic strategies. Cancer Treat Rev 2018;65:1-10. 